\title{
ONE-PARAMETER SEMIGROUPS HOLOMORPHIC AWAY FROM ZERO
}

BY

\section{MELINDA W. CERTAIN}

ABSTRACT. Suppose $T$ is a one-parameter semigroup of bounded linear operators on a Banach space, strongly continuous on $[0, \infty)$. It is known that lim sup $\operatorname{su}_{x \rightarrow 0}|T(x)-I| \leq 2$ implies $T$ is holomorphic on $(0, \infty)$. Theorem $I$ is a generalization of this as follows: Suppose $M>0,0<r<s$, and $\rho$ is in $(1,2)$. If $\left|(T(h)-I)^{n}\right| \leq M \rho^{n}$ whenever $n h$ is in $[r, s], n=1,2, \ldots, h>0$, then there exists $b>0$ such that $T$ is holomorphic on $[b, \infty)$. Theorem II shows that, in some sense, $b \rightarrow 0$ as $r \rightarrow 0$. Theorem I is an application of Theorem III: Suppose $M>0,0<r<s, \rho$ is in $(1,2)$, and $f$ is continuous on $[-4 s, 4 s]$.

If $\left|\Sigma_{q=0}^{n}\left(\begin{array}{c}n \\ q\end{array}\right)(-1)^{n-q} f(t+q h)\right| \leq M \rho^{n}$ whenever $n h$ is in $[r, s], n=1,2, \ldots$, $h>0,[t, t+n h] \subset[-4 s, 4 s]$, then $f$ has an analytic extension to an ellipse with center zero. Theorem III is a generalization of a theorem of Beurling in which the inequality on the differences is assumed for all nh. An example is given to show the hypothesis of Theorem I does not imply $T$ holomorphic on $(0, \infty)$.

1. Introduction. Suppose $T$ is a one-parameter semigroup of bounded linear operators on a Banach space. Recent work of A. Beurling [3] gives the following:

Theorem A. Suppose $T$ is weakly measurable on $(0, \infty)$. Then if $\lim \sup _{x \rightarrow 0}|T(x)-I|<2, T$ is bolomorpbic on $(0, \infty)$.

This is a generalization of a theorem due to J. Neuberger [8]:

Theorem B. Suppose $T$ is strongly continuous on $[0, \infty)$. Tben if lim $\sup _{x \rightarrow 0}|T(x)-I|<2, A T(x)$ is bounded for all $x>0$, A being the infinitesimal generator of $T$.

Under the assumption of strong continuity on $[0, \infty)$, Theorem $A$ also follows from a theorem of Kato [s].

Theorem $I$ of this note presents a generalization of Theorems $A$ and $B$ as follows: Suppose $T$ is strongly continuous on $[0, \infty)$. Suppose $M>0,0<r<s$, and $\rho$ is in $(1,2)$. If $\left|(T(b)-I)^{n}\right| \leq M \rho^{n}$ whenever $n b$ is in $[r, s], n=1,2, \ldots$, $b>0$, then there exists $b>0$ such that $T$ is holomorphic on $[b, \infty)$. An example,

Presented to the Society, January 25, 1973; received by the editors December 13, 1972 and, in revised form, May 9, 1973.

AMS (MOS) subject classifications (1970). Primary 47D05; Secondary 26A93, 39A05.

Key words and phrases. Semigroup of operators, holomorphic semigroup, analytic extension of functions, finite differences, quasianalytic classes of functions. 
due to Neuberger [9], is presented in $\$ 4$ to show that the hypothesis of Theorem I does not imply $T$ holomorphic on $(0, \infty)$. However, Theorem II says that, in some sense, $h \rightarrow 0$ as $r \rightarrow 0$.

Theorems A and B trace their beginnings, at least in part, to some earlier work of Neuberger having to do with quasianalytic classes of functions determined by conditions on finite differences. In [7] Neuberger proved the following:

Theorem C. Suppose $\rho$ and $M$ are positive numbers, $1 \leq \rho<2$, and suppose $G$ is a collection of continuous real-valued functions $f$ on $(0,1)$ such that if $u$ and $v$ are in $(0,1)$ then

$$
\left|\sum_{i=0}^{n}\left(\begin{array}{l}
n \\
i
\end{array}\right)(-1)^{n-i} f(u+i(v-u) / n)\right| \leq M \rho^{n}, \quad n=1,2, \ldots
$$

Then $G$ is a quasianalytic collection in the sense that no two members of $G$ agree on an open subinterval of $(0,1)$.

The question was raised in [7], and also by D. G. Kendall in [6] in the context of Markov semigroups, of whether $G$ could contain a nonanalytic member. Beurling has proved the following theorem which answers this question negatively.

Theorem D. Suppose $f$ is a function continuous on $[-4,4]$ and for some $M>0$ and $\rho$ in $[3 / 2,2)$,

$$
\left|\sum_{i=0}^{n}\left(\begin{array}{l}
n \\
i
\end{array}\right)(-1)^{n-i} f(u+i(v-u) / n)\right| \leq M \rho^{n}, \quad n=1,2, \ldots,
$$

$u, v$ in $[-4,4]$. Then $f$ can be extended analytically to the rhombus with vertices at $\pm 4, \pm 4 i k \alpha^{2}$ where $\alpha=(2-\rho) / 4$.

Theorems III and IV of this note generalize Theorem D in that analyticity of the function $f$ (in some open set centered at zero) is still deduced even though the inequality on the differences is assumed only for $|v-u|$ in some interval $[r, s]$, $0<r<s$. Theorem III is stated for purposes of comparison with Theorem D.

Theorem IV is a more detailed statement and includes Theorem III; consequently, a separate proof of Theorem III is not given. Theorem D is contained in [3] as a special case. The proof here of Theorem IV parallels Beurling's proof in [3] and uses, as does his proof, some techniques described in [2]. Theorem D is applied by Neuberger in [8] to prove Theorem B. Theorem I is proved from Theorem IV using some of the same techniques.

2. Definitions and statement of theorems. Suppose $X$ is a (complex) Banach space and $T$ is a one-parameter semigroup of bounded linear transformations from $X$ to $X$, strongly continuous on $[0, \infty)$. For $p$ in $X$ and $f$ in $X^{*}$, denote by $z_{p, f}$ the function on $[0, \infty)$ defined by $z_{p, f}(x)=f(T(x) p)$. 
An additive abelian semigroup (in the complex plane, in this note) will be called a semimodule. An angular semimodule is a semimodule which is an open set and which has zero as a limit point. A spinal semimodule is a semimodule which includes a ray from the origin and an open set intersected by this ray. These definitions are given in [4, pp. 256-269].

The statement that $U$ is an extension of $T$ to a domain $S$ in the complex plane means that (1) $S$ is a semimodule; (2) for $\eta$ in $S, U(\eta)$ is a bounded linear transformation from $X$ to $X$; (3) if $\lambda, \eta$ in $S$, then $U(\lambda) U(\eta)=U(\lambda+\eta)$; (4) $S \cap$ $[0, \infty)$ is not empty and if $x$ is in $S \cap[0, \infty)$ then $U(x)=T(x)$. If $U$ is an extension of $T$ to $S$, then the functions $z_{p, f}$ have an obvious extension $\widetilde{z_{p, f}}$ to $S$ : $\widetilde{z}_{p, f}(\lambda)=f\left(U(\lambda)_{p}\right), f$ in $X^{*}, p$ in $X, \lambda$ in $S$.

$U$ is said to be a holomorphic (analytic) extension of $T$ to $S$ if, for $p$ in $X$, $f$ in $X^{*}, \overparen{z_{p, f}}$ is holomorphic in $S$. Seemingly this is a definition of weak differentiability, but if $U$ is holomorphic in $S$ by the definition just given, then $U$ is continuous and differentiable in $S$ in the uniform operator topology, uniformly on compact subsets of S. For a proof and discussion see [4, pp. 92-94].

Theorem I. Suppose $r, s, \rho$ are positive numbers with $1<\rho<2, r<s$, and suppose there exists $M>0$ such that if $n$ is a nonnegative integer, $b>0$,

$$
\left|(T(b)-l)^{\prime \prime}\right| \leq M \rho^{n} \text { whenever } n=0 \text { or } n b \text { is in }[r, s] \text {. }
$$

Then there exists $b>0$ such that $T$ bas a bolomorphic extension to a domain which includes $[b, \infty)$.

Theorem II. Suppose $\rho>0$ and for each positive integer $j_{,} r_{j}$ and $s_{j}$ are numbers such that (i) $0<r_{j}<s_{j}$; (ii) $r_{j} \rightarrow 0$ as $j \rightarrow \infty$; (iii) $\left\{r_{j} / s_{j}\right\}_{j=1}^{\infty}$ is bounded away from 1. Suppose that for each positive integer $j, T_{j}$ is a strongly continuous semigroup on $[0, \infty)$ and there exists $M_{j}>0$ such that $\left|\left(T_{j}(b)-I\right)^{n}\right| \leq M_{j} \rho^{n}$ whenever $n=0$ or $n b$ is in $\left[r_{j}, s_{j}\right]$. Then there is a sequence $b_{1}, b_{2}, \cdots$ of positive numbers converging to 0 such that $T$ bas a bolomorphic extension to a domain which includes $[b, \infty)$.

Some additional notation and definitions are given before the next theorems are stated. If $\beta, \theta>0$ and $t_{0}$ is a real number, then $E_{\beta, \theta}\left(t_{0}\right)$ denotes the ellipse with foci at $t_{0}-\beta, t_{0}+\beta$ and with sum of semiaxes equal to $\beta / \theta$. $E_{\beta, \theta}(0)$ will be denoted simply $E_{\beta, \theta}$. Also $\sum_{v=0}^{n}\left(\begin{array}{c}n \\ v\end{array}\right)(-1)^{n-v} f(t+v b)$, for $f$ a function on $[t, t+n b]$, will be denoted by $\Delta_{b}^{n} f(t)$.

The statement that $f$ has an analytic extension to $E_{\beta, \theta}\left(t_{0}\right)$ means that there is a function $\tilde{f}$, analytic at every point within and on $E_{\beta, \theta}\left(t_{0}\right)$, such that if $x$ is in $\left[t_{0}-\beta, t_{0}+\beta\right], \tilde{f}(x)=f(x)$. 
Theorem III. Suppose $r, s$ are positive numbers, $r<s, f$ is a function continuous on $[-4 s, 4 s]$, and for some $M>0, \rho$ in $(1,2)$,

$$
\left|\sum_{q=0}^{n}\left(\begin{array}{l}
n \\
q
\end{array}\right)(-1)^{n-q} f(u+q(v-u) / n)\right| \leq M \rho^{n}
$$

if $u, v$ are in $[-4 s, 4 s],|v-u|$ is in $[r, s], n=1,2, \ldots$. Then if $\sigma$ is in $(\rho, 2)$ there exists a number $\beta, 0<\beta<\sigma(s-r) / 8$, sucb that $f$ can be extended analytically to the ellipse $E_{\beta, \sigma / 2}$.

Theorem IV. Suppose $r, s, \rho$ are positive numbers with $1<\rho<2, r<s$. Then there are positive numbers $D, \beta, \sigma$ such that the following is true: Suppose $K>0$ and denote by $G_{K}$ a collection of functions $f$ such that for some real number $t_{0}$,

(1) $f$ is continuous on $\left[t_{0}-D, t_{0}+D\right]$, and

(2) $\left|\Delta_{b}^{n} f(t)\right| \leq K \rho^{n}$, whenever $n=0$ or $n b$ is in $[r, s]$ and $[t, t+n b] C$ $\left[t_{0}-D, t_{0}+D\right]$.

Then there exists $\tilde{K}>0$ such that if $f$ is in $G_{K}$, $f$ continuous on $\left[t_{0}-D, t_{0}+D\right]$, then $f$ bas an analytic extension $\tilde{f}$ to $E_{\beta_{0} \sigma / 2}\left(t_{0}\right)$ and $\tilde{f}$ is bounded by $\widetilde{K}$ in $E_{\beta, \sigma / 2}\left(t_{0}\right)$.

3. Proofs. The proof of Theorem IV is given first. It depends upon the following theorem of S. Bernstein [1, p. 112]:

Theorem $\mathbf{E}$. Suppose $f$ is a function continuous on $[-\beta, \beta]$ and there exist polynomials $P_{n}$ of degree $n, \theta_{0}$ in $(0,1)$, and $M>0$ such that

$$
\int_{-\beta}^{\beta}\left|f(t)-P_{n}(t)\right|^{2} d t<M \theta_{0}^{2 n}, \quad n=0,1,2, \ldots
$$

Then if $\theta$ is in $\left(\theta_{0}, 1\right), f$ bas an analytic extension $\tilde{f}$ to $E_{\beta, \theta}$. Furthermore, if $M, \beta>0$ and $0<\theta_{0}<\theta<1$, there exists $\tilde{M}$ sucb that for any continuous function $f$ for which there exist polynomials $P_{n}$ of degree $n$ sucb that (3) bolds, the extension $\tilde{f}$ is bounded by $\tilde{M}$ in $E_{\beta, \theta}$.

Lemma. If $r_{0}, \delta_{0}>0, n$ is a positive integer, and $|x| \geq 4 \pi n / \delta_{0}$, then

$$
\int_{r_{0} / n}^{\left(r_{0}+\delta_{0}\right) / n} \sin ^{2 n}(b x / 2) d b \geq \delta_{0} / 4 n^{2} .
$$

Proof of lemma. Suppose $n$ is a positive integer and $x \geq 4 \pi n / \delta_{0}$. Then there is a positive integer $K \geq 1$ such that $x$ is in $\left[4 \pi n K / \delta_{0}, 4 \pi n(K+1) / \delta_{0}\right]$. It is easy to verify that $\int_{0}^{2 K \pi} \sin ^{2 n} u d u \geq K \pi / n$. Hence, one has 


$$
\begin{aligned}
\int_{r_{0} / n}^{\left(r_{0}+\delta_{0}\right) / n} \sin ^{2 n}(b x / 2) d b & =2 / x \int_{r_{0} x / 2 n}^{\left(r_{0}+\delta_{0}\right) x / 2 n} \sin ^{2 n} u d u \\
& \geq 2 / x \int_{0}^{2 K \pi} \sin ^{2 n} u d u \geq 2 K \pi / x n
\end{aligned}
$$

using that $x \geq 4 n K \pi / \delta_{0}$ and hence $\delta_{0} x / 2 n \geq 2 K \pi$. But also $x \leq 4 \pi n(K+1) / \delta_{0}$ and hence $2 K \pi / x n \geq \delta_{0} K / 2 n^{2}(K+1) \geq \delta_{0} / 4 n^{2}$.

Proof of Theorem IV. Suppose r, $s, \rho$ are positive numbers with $1<\rho<2$, $r<s$.

Choose $\sigma$ such that $\rho<\sigma<2$.

The choice of $D$ and $\beta$ is more complicated but an explicit procedure follows.

Choose $\sigma_{0}$ such that $\rho<\sigma_{0}<\sigma$, choose $a$ such that

(i) $0<a<1 / 2$,

(ii) $a<1-(r / s)$, and

(iii) for all positive integers $n$,

$$
\left(\begin{array}{c}
n \\
{[a n]}
\end{array}\right) \rho^{n}<\sigma_{0}^{n}
$$

choose $B>0$ such that $(s / B)^{\alpha}<\sigma_{0} / 4$. Denote $r /(1-\alpha)$ by $r_{0}$ and denote $s-r_{0}$ by $\delta_{0}$. Then let $D=3 B+s$ and $\beta=\sigma_{0} \delta_{0} / 8 e \pi$.

Suppose $K>0$ and denote by $G_{K}$ a collection of functions as described in the statement of the theorem. The selection of $\widetilde{K}$ is made as follows: Denote by $\sigma_{1}$ a number such that $\sigma_{0}<\sigma_{1}<\sigma$, by $K_{0}$ a number such that

$$
K_{0} \geq \max \left\{24 \sqrt{2 \pi} B K / \delta_{0}, 16 K^{2}(6 B+s)\right\},
$$

and by $K_{1}$ a number such that

$$
2 \beta\left(K_{0}\right)^{2}\left(\sigma_{0} / 2\right)^{2 n}+n^{3} K_{0}\left(\sigma_{0} / 2\right)^{2 n}<K_{1}\left(\sigma_{1} / 2\right)^{2 n}, \quad n=1,2, \ldots
$$

Choose $\widetilde{K}$ to be a number such that if $f$ is continuous on $[-\beta, \beta]$ and, for some polynomials $P_{n}$, (3) holds with $M$ replaced by $K_{1}$ and $\theta_{0}$ replaced by $\sigma_{1} / 2$, then $f$ has an analytic extension $\tilde{f}$ to $E_{\beta, \sigma / 2}$ and $\tilde{f}$ is bounded by $\tilde{K}$ on $E_{\beta, \sigma / 2}$. The theorem of Bernstein quoted above says this is possible.

Suppose now that $f$ is a member of $G_{K}$. Then $f$ is continuous on $\left[t_{0}-D, t_{0}+D\right]$ for some real number $t_{0}$. It can be assumed that $t_{0}=0$. The essence of the proof is the construction of polynomials $P_{n}$ which approximate $f$ on $[-\beta, \beta]$ in such a way that Bernstein's theorem can be invoked.

The first step is to replace $f$ by functions $f_{n}$ which coincide with $f$ on $[-B, B]$ and vanish off $[-3 B, 3 B]$. 
The norms of $L^{1}(-\infty, \infty), L^{2}(-\infty, \infty)$, and $L^{\infty}(-\infty, \infty)$ will be denoted $\|\cdot\|_{1}$, $\|\cdot\|_{2},\|\cdot\|_{\infty}$, respectively. Also, if $g$ is a function, $n$ a nonnegative integer, and $t$ a number, then $g^{(n)}(t)$ denotes the $n$th derivative of $g$ at $t$.

For $n$ a positive integer, define $Q_{n, B}$ by

$$
Q_{n, B}(t)= \begin{cases}B^{-2 n}\left(B^{2}-t^{2}\right)^{n}, & \text { if }|t| \leq B ; \\ 0, & \text { if }|t|>B .\end{cases}
$$

An important property of $Q_{n, B}$ is that

$$
\left|Q_{n, B}^{(m)}(t)\right| \leq(2 n / B)^{n}, \quad n=0,1, \cdots, n-1 \text {, all real } t .
$$

To verify this suppose $t$ is in $(-B, B)$. Then, for any positive integer $n$, $\left|Q_{n, B}^{(m)}(t)\right| \leq(2 n / B)^{m}$ for all nonnegative integers $m$ : use induction on $n$ and the fact that if $f, g$ each possess $m$ derivatives at $t$ then

$$
(f g)^{(m)}(t)=\sum_{v=0}^{m}\left(\begin{array}{l}
m \\
v
\end{array}\right) f^{(v)}(t) g^{(m-v)}(t) .
$$

If $|t|>B$ then $Q_{n}^{(m)}(t)=0$ for all nonnegative integers $m$. Finally, if $t= \pm B$, an elementary argument gives $Q_{n, B}^{(m)}(t)=0$ for $m=0,1,2, \ldots, n-1$.

Denote $\int_{-\infty}^{\infty} Q_{n, B}(t) d t$ by $\gamma_{n, B}$.

Then $\gamma_{n, B}=2 B(2 \cdot 4 \cdot 6 \ldots(2 n)) /(3 \cdot 5 \cdot 7 \ldots(2 n+1))\left[2\right.$, p. 4] and $\gamma_{n, B}$ $>B / \sqrt{n}$ for all positive integers $n$.

As in [2, p. 4] and [3, p. 392], multiplier functions are now defined.

Define $k_{n, B}(t)=\gamma_{n}^{-1} \int_{-\infty}^{t}\left(Q_{n, B}(u+2 B)-Q_{n, B}(u-2 B)\right) d u$.

Using (4) one has

$$
\left|k_{n, B}^{(v)}(t)\right| \leq \gamma_{n}^{-1}(2 n / B)^{v-1} \leq(\sqrt{n} / B)(2 n / B)^{v-1},
$$

$v=1,2, \cdots, n, t$ any real number.

If a function $g$ has $v$ continuous derivatives on $[t, t+v b]$, then there exists a number $c$ in $[t, t+v b]$ such that $\left(\Delta_{b}^{v} g\right)(t)=b^{v} g^{(v)}(c)$.

This together with (5) gives the following:

$$
\left|\Delta_{b}^{v} k_{n, B}(t)\right|<b^{v}(\sqrt{n} / B)(2 n / B)^{\nu-1}=(\sqrt{n} / B)(2 b n / B)^{v-1} b, \quad v=1,2, \cdots, n ;
$$

hence,

$$
\left|\Delta_{b}^{v} k_{n, B}(t)\right| \leq(2 b n / B)^{v}, \quad v=0,1,2, \cdots, n .
$$

Now for each positive integer $n$, define $f_{n}$ by 


$$
f_{n}(t)= \begin{cases}\left(f k_{n, B}\right)(t), & \text { if }|t| \leq D \\ 0, & \text { if }|t|>D\end{cases}
$$

Then for each positive integer $n$,

(i) $f_{n}$ is continuous on $(-\infty, \infty)$;

(ii) $f_{n}$ has its support in $[-3 B, 3 B]$ and agrees with $f$ on $[-B, B]$;

(iii) $\left\|f_{n}\right\|_{1}<6 B K$.

The next step is to define polynomials $P_{n}$, of degree $n$, such that (3) holds with $M$ replaced by $K_{1}$ and $\theta_{0}$ replaced by $\sigma_{1} / 2$.

Denote by $\hat{f}_{n}$ the Fourier transform of $f_{n}$. For $n=1,2, \ldots$, define

$$
P_{n}(t)=\frac{1}{\sqrt{2 \pi}} \int_{|x|<4 \pi n / \delta_{0}}\left(\hat{f}_{n}(x) \sum_{v=0}^{n}(i t x)^{v} / v !\right) d x .
$$

If $\hat{f}_{n}$ denotes the Fourier transform of $f_{n}$, then the Fourier transform of $\Delta_{b}^{n} f_{n}$ at $x$ is $\hat{f}_{n}(x)\left(e^{i b x}-1\right)^{n}$. By Parseval's relation

$$
\int_{-\infty}^{\infty}\left|\hat{f}_{n}(x)\right|^{2}\left|e^{i b x}-1\right|^{2 n} d x
$$

$$
=\int_{-\infty}^{\infty}\left|\Delta_{b}^{n} f_{n}(x)\right|^{2} d x, \quad n=1,2, \ldots, b>0
$$

In what follows, a bound on $\left|\Delta_{b}^{n} f_{n}\right|$ is deduced for $n b$ in $[r /(1-a), s]=\left[r_{0}, s\right]$. Suppose $t$ is a real number, $n$ is a positive integer, $b>0$, and $g_{1}, g_{2}$ are functions each of whose domain includes $[t, t+n b]$. Then

$$
\left(\Delta_{b}^{n} g_{1} g_{2}\right)(t)=\sum_{v=0}^{n}\left(\begin{array}{l}
n \\
v
\end{array}\right)\left(\Delta_{b}^{n-v} g_{1}\right)(t)\left(\Delta_{b}^{v} g_{2}\right)(t+(n-v) b) .
$$

From (9),

$$
\begin{aligned}
\left|\Delta_{b}^{n} f_{n}(t)\right|< & \sum_{v=0}^{\left[a_{n}\right]}\left(\begin{array}{l}
n \\
v
\end{array}\right)\left|\Delta_{b}^{n-v} f(t) \| \Delta_{b}^{v} k_{n}(t+(n-v) b)\right| \\
& +\sum_{v=\left[a_{n}\right]_{+1}}^{n}\left(\begin{array}{l}
n \\
v
\end{array}\right)\left|\Delta_{b}^{n-v_{f}}(t) \| \Delta_{b}^{v} k_{n}(t+(n-v) b)\right| .
\end{aligned}
$$

Suppose $n b$ is in $\left[r_{0}, s\right]$. Then if $t$ is outside $[-3 B-s, 3 B], \Delta_{b}^{n} f_{n}(t)=0$. Suppose $t$ is in $[-3 B-s, 3 B]$. Since $n b$ is in $\left[r_{0}, s\right]$, if $v \leq\left[\alpha_{n}\right] \leq a_{n}$, then $(n-v) b$ is in $[r, s]$ and hence $\left|\Delta_{b}^{n-v} f(t)\right| \leq K \rho^{n-v}$. Also $2 b n / B<1$, since $(s / B)^{a}<\sigma_{0} / 4<1 / 2$. Hence, 


$$
\begin{aligned}
\sum_{v=0}^{\left[a_{n}\right]}\left(\begin{array}{l}
n \\
v
\end{array}\right) \mid \Delta_{b}^{n-v} f(t) \| \Delta_{b}^{v} k_{n}(t+(n-v) b \mid \\
\leq \sum_{v=0}^{\left[a_{n}\right]}\left(\begin{array}{l}
n \\
v
\end{array}\right) K \rho^{n-v}(2 b n / B)^{v} \leq n K\left(\begin{array}{c}
n \\
{\left[a_{n}\right]}
\end{array}\right) \rho^{n} \\
<n K \sigma_{0}^{n}, \quad \text { using also that } a<1 / 2 \text { and } \rho>1 .
\end{aligned}
$$

Any function $g$ bounded by $M$ on $[t, t+n b]$ satisfies $\left|\Delta_{b}^{n} g(t)\right| \leq M 2^{n}, n=$ $1,2, \ldots, b>0$. Hence for $n b$ in $\left[r_{0}, s\right]$ and $t$ a real number,

$$
\begin{aligned}
\sum_{v=\left[a_{n}\right]_{+1}}^{n}\left(\begin{array}{l}
n \\
v
\end{array}\right)\left|\Delta_{b}^{n-v} f(t)\right|\left|\Delta_{b}^{v} k_{n}(t+(n-v) b)\right| \\
\leq \sum_{v=\left[a_{n}\right]_{+1}}^{n}\left(\begin{array}{l}
n \\
v
\end{array}\right) K 2^{n-v}(2 b n / B)^{v} \\
\leq 2^{n} K \sum_{v=\left[a_{n}\right]_{+1}}^{n}\left(\begin{array}{l}
n \\
v
\end{array}\right)(s / B)^{v}<4^{n} K(s / B)^{a n}<K \sigma_{0}^{n} .
\end{aligned}
$$

Thus if $n b$ is in $\left[r_{0}, s\right],(10)$ and (11) give $\left|\Delta_{b}^{n} f_{n}\right| \leq 2 K_{n}\left(\sigma_{0}\right)^{n}, n=1,2, \ldots$. Since $\Delta_{b}^{n} f_{n}$ is zero outside $[-3 B-s, 3 B]$, using (8) one gets

$$
\begin{aligned}
\int_{-\infty}^{\infty}\left|\hat{f}_{n}(x)\right|^{2} 2^{2 n} \sin ^{2 n}(b x / 2) d x & =\int_{-\infty}^{\infty}\left|\hat{f}_{n}(x)\right|^{2}\left|e^{i b x}-1\right|^{2 n} d x \\
& <(6 B+s)\left(2 K n\left(\sigma_{0}\right)^{n}\right)^{2}, \text { if } n b \text { in }\left[r_{0}, s\right]
\end{aligned}
$$

Hence

$$
\begin{aligned}
\int_{\left[r_{0} / n, s / n\right]}\left(\int_{|x| \geq 4 \pi n / \delta_{0}}\left|\hat{f}_{n}(x)\right|^{2} \sin ^{2 n}(b x / 2) d x\right) d b & \\
& <n \delta_{0}(6 B+s) 4 K^{2}\left(\sigma_{0} / 2\right)^{2 n}, \quad n=1,2, \ldots
\end{aligned}
$$

Using the lemma stated above and reversing the order of integration one has

$$
\begin{aligned}
\int_{|x| \geq 4 \pi n / \delta_{0}}\left|\hat{f}_{n}(x)\right|^{2} d x & <4 n^{3}(6 B+s) 4 K^{2}\left(\sigma_{0} / 2\right)^{2 n} \\
& <K_{0} n^{3}\left(\sigma_{0} / 2\right)^{2 n}, \quad n=1,2, \ldots
\end{aligned}
$$

Define $g_{n}(t)=1 / \sqrt{2 \pi} \int_{|x|<4 \pi n / \delta_{0}}\left(\hat{f}_{n}(x) e^{i t x}\right) d x, n=1,2, \ldots$. If for $n$ a positive integer,

$$
b_{n}(t)= \begin{cases}\hat{f}_{n}(t), & |t|<4 \pi n / \delta_{0} \\ 0, & |t| \geq 4 \pi n^{\prime} \delta_{0}\end{cases}
$$


then $g_{n}(t)=\int_{-\infty}^{\infty} b_{n}(x) e^{i t x} d x$, so $b_{n}=\hat{g}_{n}$ in $L^{2}(-\infty, \infty)$. Hence

$$
\left\|\hat{f}_{n}-\hat{g}_{n}\right\|_{2}=\left\|\hat{f}_{n}-b_{n}\right\|_{2}=\int_{|x| \geq 4 \pi n / \delta_{0}}\left|\hat{f}_{n}(x)\right|^{2} d x<K_{0} n^{3}\left(\sigma_{0} / 2\right)^{2 n}
$$

also

$$
\begin{aligned}
& \int_{-\beta}^{\beta}\left|f(t)-g_{n}(t)\right|^{2} d t<\int_{-B}^{B}\left|f(t)-g_{n}(t)\right|^{2} d t \\
& \quad=\int_{-B}^{B}\left|f_{n}(t)-g_{n}(t)\right|^{2} d t \leq\left\|f_{n}-g_{n}\right\|_{2}=\left\|\hat{f}_{n}-\hat{g}_{n}\right\|_{2}
\end{aligned}
$$

which yields

$$
\int_{-\beta}^{\beta}\left|f(t)-g_{n}(t)\right|^{2} d t<K_{0} n^{3}\left(\sigma_{0} / 2\right)^{2 n}, \quad n=1,2, \ldots
$$

Suppose $t$ is a real number and $n$ is a positive integer. Then

$$
\begin{aligned}
\left|g_{n}(t)-P_{n}(t)\right| & \leq \frac{1}{\sqrt{2 \pi}} \int_{|x|<4 \pi n / \delta_{0}}\left|\hat{f}_{n}(x)\right||t x|^{n+1} /(n+1) ! d x \\
& \leq\left\|\hat{f}_{n}\right\|_{\infty}|t|^{n+1} / \sqrt{2 \pi}(n+1) ! \int_{|x|<4 \pi n / \delta_{0}}|x|^{n+1} d x \\
& \leq\left(4 \pi n / \delta_{0}\right)^{n+2}\left(2\left\|f_{n}\right\|_{1}|t|^{n+1} / \sqrt{2 \pi}(n+1) !(n+2)\right),
\end{aligned}
$$

using that $\left|e^{i t x}-\sum_{v=0}^{n}(i t x)^{v} / v !\right| \leq|t x|^{n+1} /(n+1) !$ and that $n !>(n / e)^{n}$.

If $|t| \leq \beta=\sigma_{0} \delta_{0} / 8 e \pi$, then

$$
\begin{aligned}
\left|g_{n}(t)-P_{n}(t)\right| & <\left(48 B K \pi / \sqrt{2 \pi} \delta_{0}\right)(n /(n+2))(n /(n+1))^{n+1}\left(\sigma_{0} / 2\right)^{n+1} \\
& <K_{0}\left(\sigma_{0} / 2\right)^{n}, \quad n=1,2, \ldots
\end{aligned}
$$

Hence,

$$
\int_{-\beta}^{\beta}\left|g_{n}(t)-P_{n}(t)\right|^{2} d t<2 \beta K_{0}^{2}\left(\sigma_{0} / 2\right)^{2 n}
$$

Recalling that $K_{1}$ is a number such that $2 \beta\left(K_{0}\right)^{2}\left(\sigma_{0} / 2\right)^{2 n}+n^{3} K_{0}\left(\sigma_{0} / 2\right)^{2 n}<$ $K_{1}\left(\sigma_{1} / 2\right)^{2 n}, n=1,2, \ldots$, one gets, from (12) and (13),

$$
\int_{-\beta}^{\beta}\left|f(t)-P_{n}(t)\right|^{2} d t<K_{1}\left(\sigma_{1} / 2\right)^{2 n}, \quad n=1,2, \ldots,
$$

and the desired analytic extension of $f$ follows from Bernstein's theorem.

Proof of Theorem I. Suppose $r, s, \rho$ are positive numbers with $1<\rho<2$, $r<s$, and suppose $M$ is a number such that if $n$ is a nonnegative integer, $b>0$, and $n b$ is in $[r, s]$, then $\left|(T(b)-I)^{n}\right|<M \rho^{n}$. Suppose $D, \beta, \sigma$ are positive numbers such that Theorem IV holds for $r, s, \rho, D, \beta, \sigma$. The claim is that the conclusion of Theorem I holds for $b=D-\beta$. 
Suppose $t \geq 0$. It is easy to verify that for $b>0, n$ a nonnegative integer, $p$ in $X$, and $f$ in $X^{*},\left|\Delta_{b}^{n} z_{p, f}(t)\right| \leq|f|\|p\||T(t)|\left|(T(b)-l)^{n}\right|$. Denote by $M_{0}$ a number such that $|T(t)| \leq M_{0}$ if $t$ is in $[0,2 D]$. If $n b$ is in $[r, s],\|p\| \leq 1,|f| \leq 1$, then $\left|\Delta_{b}^{n} z, f(t)\right| \leq M_{0} M \rho^{n}$ if $[t, t+n b] \subset[0,2 D]$. Hence, by Theorem IV, there exists $\widetilde{M}$ such that if $\|p\| \leq 1,|f| \leq 1, z_{p, f}$ has an analytic extension $z_{p, f}$ to $E_{\beta, \sigma / 2}(D)$ and $\widetilde{z_{p, f}}$ is bounded by $\tilde{M}$ in $E_{\beta, \sigma / 2}(D)$.

Denote by $B(x ; \epsilon)$ the ball in the complex plane with center at $x$ and radius $\epsilon, x$ a real number, $\epsilon>0$.

The ellipse $E_{\beta, \sigma / 2}(D)$ has its foci at $D-\beta, D+\beta$. Hence there exists $\delta>0$ such that $B(b ; 2 \delta)=B(D-\beta ; 2 \delta)$ is contained in $E_{\beta, \sigma / 2}(D)$. Since $\overbrace{p, f}$ is bounded by $\tilde{M}$ in $E_{\beta, \sigma / 2}(D),\|p\| \leq 1,|f| \leq 1$, then if $\lambda$ is in $B(b ; \delta)$,

$$
\widetilde{z_{p, f}^{(n)}}(\lambda) \mid \leq n ! \tilde{M} \delta^{-n}, \quad n=0,1,2, \ldots
$$

The claim is that if $t$ is in $B(b ; \delta)$, then $A^{n} T(t)$ is a bounded operator on $X$, $\left\|A^{n} T(t)\right\| \leq n ! \tilde{M} \delta^{-n}, n=0,1,2, \ldots$. The argument verifying this, by induction on $n$, is presented below; for the case $n=1$, it is found in [8] of Neuberger.

If $n=0$, the claim is obviously true since $\left|z_{p, f}(t)\right| \leq \tilde{M},\|p\| \leq 1,|f| \leq 1, t$ in $B(b ; \delta)$, implies $\|T(t)\| \leq \widetilde{M}, t$ in $B(b ; \delta)$.

Suppose $K$ is a positive integer and suppose $A^{K-1} T(t)$ is a bounded operator on $X, t$ in $B(b ; \delta)$. It will be shown that $A^{K} T(t)$ is a bounded operator on $X$, for $t$ in $B(b ; \delta)$, and that $\left\|A^{K} T(t)\right\| \leq K ! \tilde{M} \delta^{-K}$.

Suppose $t$ is in $B(b ; \delta)$ and $p$ is in the domain of $A$. Then $A^{K} T(t) p=$ $\lim _{x \rightarrow t^{+}}(x-t)^{-1}(T(x)-I) A^{K-1} T(t) p$, if this limit exists. By assumption, $A^{K-1} T(x) p$ exists for all $x$ in $B(b ; \delta)$. Then

$$
\begin{aligned}
& \lim _{x \rightarrow t^{+}}(x-t)^{-1}(T(x-t)-I) A^{K-1} T(t) p \\
& \quad=\lim _{x \rightarrow t^{+}}(x-t)^{-1}\left(T(x-t) A^{K-1} T(t) p-A^{K-1} T(t) p\right) \\
& =\lim _{x \rightarrow t^{+}}(x-t)^{-1}\left(A^{K-1} T(x) p-A^{K-1} T(t) p\right) \\
& =\lim _{x \rightarrow t^{+}} A^{K-1} T(t)\left((x-t)^{-1}(T(x-t)-I)\right) p \\
& =A^{K-1} T(t)\left(\lim _{x \rightarrow t^{+}}(x-t)^{-1}(T(x-t)-I) p\right)=A^{K-1} T(t) A p,
\end{aligned}
$$

and thus $A^{K} T(t) p$ exists. The above equalities also show that if $p$ is any point of $X$ and $\lim _{x \rightarrow t^{+}}(x-t)^{-1}\left(A^{K-1} T(x) p-A^{K-1} T(t) p\right)$ exists, then this limit is $A^{K} T(t) p$. 
Suppose $f$ is in $X^{*}, p$ is in $X,\|f\| \leq 1,\|p\| \leq 1$. Then

$$
\begin{aligned}
& \left|f\left((x-t)^{-1}\left(A^{K-1} T(x)-A^{K-1} T(t)\right) p\right)\right| \\
& \quad=\left|(x-t)^{-1}\left(z_{p, f}^{(K-1)}(x)-z_{p, f}^{(K-1)}(t)\right)\right|=\left|z_{p, f}^{(K)}\left(x_{0}\right)\right|
\end{aligned}
$$

for some $x_{0}$ in $[x, t]$, and $\left|z_{p, f}^{(K)}\left(x_{0}\right)\right| \leq \delta^{-K} K ! \tilde{M}$ if $[x, t]$ is in $B(b ; \delta)$.

Hence if $[x, t]$ is in $B(b ; \delta)$,

$$
\left\|(x-t)^{-1}\left(A^{K-1} T(x)-A^{K-1} T(t)\right)\right\| \leq \delta^{-K} K ! \tilde{M} \text {. }
$$

Thus if $t$ is in $B(b ; \delta), \lim _{x \rightarrow t^{+}}(x-t)^{-1}\left(A^{K-1} T(x) p-A^{K-1} T(t) p\right)$ exists for $p$ in a dense set (the domain of $A$ ), and also $\left\|(x-t)^{-1}\left(A^{K-1} T(x)-A^{K-1} T(t)\right)\right\|$ $\leq \delta^{-K} K ! \tilde{M}$ when $[x, t]$ is in $B(b ; \delta)$.

Hence for any $p$ in $X, t$ in $B(b ; \delta), \lim _{x \rightarrow t^{+}}(x-t)^{-1}\left(A^{K-1} T(x) p-A^{K-1} T(t) p\right)$ exists, this limit is $A^{K} T(t) p$, and $\left\|A^{K} T(t)\right\| \leq \delta^{-K} K ! \tilde{M}$.

Suppose $\lambda$ is in $B(b ; \delta / 2)$. Then $W(\lambda) p=\sum_{n=0}^{\infty}\left((\lambda-b)^{n} / n !\right) A^{n} T(b) p$ defines $W(\lambda)$ as a bounded linear transformation on $X$. Furthermore, $W$ is holomorphic at each $\lambda$ in $B(b ; \delta / 2)$ since if $f$ is in $X^{*}, p$ in $X$, then

$$
\begin{aligned}
f(W(\lambda) p) & =\sum_{n=0}^{\infty}\left((\lambda-b)^{n} / n !\right) f\left(A^{n} T(b) p\right) \\
& =\sum_{n=0}^{\infty}\left((\lambda-b)^{n} / n !\right) z_{p, f}^{(n)}(b)=\widetilde{z_{p, f}(\lambda)}
\end{aligned}
$$

and $\overparen{z_{p, f}}$ is holomorphic at $\lambda$.

Thus there is a function $W$ from $B(b ; \delta / 2)$ to the set of bounded linear transformations on $X, W$ is holomorphic at each $\lambda$ in $B(b ; \delta / 2)$, and if $x$ is in $(b-(\delta / 2), b+(\delta / 2))$, then $W(x)=T(x)$. By a theorem of Hille [4, p. 477] $T$ has an analytic extension to the interior of a spinal semimodule which includes $[b, \infty)$.

Proof of Theorem II. Suppose $\rho$ is a number, $1<\rho<2$, and $\left\{\left[r_{j}, s_{j}\right]\right\}_{j=1}^{\infty}$ is a sequence of intervals such that $r_{j} \rightarrow 0$ as $j \rightarrow \infty$ and such that there exists $\epsilon>0$ such that $r_{j} / s_{j}<1-\epsilon, j=1,2, \ldots$. Suppose that for each $j, T_{j}$ is a strongly continuous semigroup on $[0, \infty)$ and there exists $M_{j}>0$ such that if $n$ is a nonnegative integer, $b>0$, and $n=0$ or $n b$ is in $\left[r_{j}, s_{j}\right]$, then $\left|\left(T_{j}(b)-I\right)^{n}\right| \leq$ $M_{j} \rho^{n}$.

Denote $r_{j} /(1-\epsilon)$ by $s_{j}^{\prime}$. Then $r_{j}<s_{j}^{\prime}<s_{j}$, for all $j$, and $s_{j}^{\prime} \rightarrow 0$ as $j \rightarrow \infty$. Suppose $\sigma, \sigma_{0}$ are numbers such that $\rho<\sigma_{0}<\sigma<2$, and suppose $\alpha$ is a number such that $\alpha<\epsilon, \alpha$ is in $(0,1 / 2)$, and

$$
\left(\begin{array}{c}
n \\
{\left[\alpha_{n}\right]}
\end{array}\right) \rho^{n}<\sigma_{0}^{n}
$$

$n=1,2, \ldots$. Then $a<1-\left(r_{j} / s_{j}^{\prime}\right)=\epsilon, j=1,2, \ldots$. 
Since $s_{j}^{\prime} \rightarrow 0$ as $j \rightarrow \infty$, there exists a sequence of positive numbers $\left\{B_{j}\right\}_{j=1}^{\infty}$ such that $B_{j} \rightarrow 0$ as $j \rightarrow \infty$ and such that $\left(s_{j}^{\prime} / B_{j}\right)^{a}<\sigma_{0} / 4, j=1,2, \ldots$. Denote $r_{j} /(1-a)$ by $r_{0, j}$ and denote $s_{j}^{\prime}-r_{0, j}$ by $\delta_{0, j}$. Let $D_{j}=3 B_{j}+s_{j}^{\prime}$ and let $\beta_{j}=$ $\delta_{0, j} \sigma_{0} / 8 e \pi$. Then Theorem IV holds for $r_{j}, s_{j}^{\prime}, \rho, D_{j}, \beta_{j}, \sigma$, and hence for $r_{j}, s_{j}$, $\rho, D_{j}, \beta_{j}, \sigma$ since $\left[r_{j}, s_{j}\right] \subset\left[r_{j}, s_{j}\right]$. Let $b_{j}=D_{j}-\beta_{j}, j=1,2, \ldots$. Then for each $j$, Theorem I holds for $T_{j}, r_{j}, s_{j}, \rho$, and $b_{j}$. Clearly $D_{j} \rightarrow 0$ as $j \rightarrow \infty$. Hence $b_{j} \rightarrow 0$ as $j \rightarrow \infty$.

4. Example. The following example is due to Neuberger [9]. Suppose $X=$ $C_{[0,1] ; 0}$, the space of all functions $b$ continuous on $[0,1]$, with $b(0)=0$, and with $\|b\|=\sup _{x \in[0,1]}\{|b(x)|\}$.

For each $\lambda \geq 0$, define

$$
(T(\lambda) b)(x)= \begin{cases}0 & \text { if } \lambda-x \geq 0, \\ b(x-\lambda) & \text { if } x-\lambda \geq 0,\end{cases}
$$

$x$ in $[0,1], b$ in $C_{[0,1] ; 0}$.

Then $T$ is a one-parameter semigroup of operators on $C_{[0,1] ; 0} T$ is strangly continuous at $\lambda>1$ since $T(\lambda)=0$ for all $\lambda>1 ; T$ is strongly continuous at $\lambda<1$ since each element of $C_{[0,1] ; 0}$ is uniformly continuous on $[0,1]$; and $T$ is strongly continuous at $\lambda=1$ since each element $b$ of $C_{[0,1] ; 0}$ is continuous at 0 and $b(0)=0$.

Suppose $a$ is a number such that $0<a<1 / 2$. Then there exists $M>0$ and $\rho$ in $(1,2)$ such that $\sum_{v=0}^{\left[a_{n}\right]}\left(\begin{array}{l}n \\ v\end{array}\right)<M \rho^{n}, n=0,1,2, \ldots$. Denote $1 / \alpha$ by $r$, and suppose $s$ is any number $>r$. Then if $n \lambda$ is in $[r, s],\left|(T(\lambda)-I)^{n}\right|<M \rho^{n}, n=0$, $1,2, \ldots$, since

$$
\begin{aligned}
\left\|(T(\lambda)-I)^{n} b\right\|= & \left\|\sum_{v=0}^{n}\left(\begin{array}{l}
n \\
v
\end{array}\right)(-1)^{n-v} T(v \lambda) b\right\| \\
\leq & \left\|\sum_{v=0}^{\left[a_{n}\right]}\left(\begin{array}{l}
n \\
v
\end{array}\right)(-1)^{n-v} T(v \lambda) b\right\| \\
& +\left\|\sum_{v=\left[\begin{array}{l}
a_{n} \\
0
\end{array}+1\right.}^{n}\left(\begin{array}{l}
n \\
v
\end{array}\right)(-1)^{n-v} T(v \lambda) b\right\| \\
& \leq\|b\| \sum_{v=0}^{a_{n}}\left(\begin{array}{l}
n \\
v
\end{array}\right) \leq M \rho^{n}\|b\|,
\end{aligned}
$$

using that $v \lambda \geq a_{n \lambda}>1$ implies $\underset{v=\left[a_{n}\right]+1}{\left[a_{n}\right]}\left(\begin{array}{c}n \\ v\end{array}\right)(-1)^{n-v} T(v \lambda) b=0$. 
However, $T$ does not have an analytic extension to an open set which has zero as a limit point. Suppose $t_{0}$ is in $(0,1)$, suppose $g(x)=x, x$ in $[0,1]$, and suppose $f_{t_{0}}(b)=b\left(t_{0}\right), b$ in $C_{[0,1] ; 0}$. Then the function $z_{8, f_{t_{0}}}$, where $z_{8, f_{t_{0}}}(\lambda)=$ $f_{t_{0}}(T(\lambda) g)$, is not analytic at $t_{0}$ since

$$
z_{g, f_{t_{0}}}(x)= \begin{cases}t_{0}-x & \text { if } t_{0}-x \geq 0 \\ 0 & \text { if } x-t_{0} \geq 0\end{cases}
$$

\section{REFERENCES}

1. S. Bemstein, Leçons sur les propriétés extrémales et la meilleure approximation des fonctions analytiques d'une variable réelle, Gauthiers-Villars, Paris, 1926.

2. A. Beurling, On quasi-analyticity and general distributions, Multigraphed lecture notes, Stan ford University, Stanford, Calif., 1961, Lecture 3.

3. - On analytic extension of semigroups of operators, J. Functional Analysis 6 (1970), 387-400. MR $43 \# 7960$.

4. E. Hille and R. S. Phillips, Functional analys is and semi-groups, rev. ed., Amer. Math. Soc. Colloq. Publ., vol. 31, Amer. Math. Soc., Providence, R. I., 1957. MR 19, 664 .

5. T. Kato, A characterization of holomorphic semigroups, Proc. Amer. Math. Soc. 25 (1970), 495-498. MR 41 \#9050.

6. D. G. Kendall, Some recent developments in the theory of denumerable Markov processes, Tran's. Fourth Prague Conf. on Information Theory, Statistical Decision Functions, Random Processes (Prague, 1965), Academia, Prague, 1967, pp. 11-27.

MR 36 \#974.

7. J. W. Neuberger, A quasi-analyticity condition in terms of finite differences, Proc. London Math. Soc. (3) 14 (1964), 245-259. MR 28 \#3130.

8. - Analyticity and quasi-analyticity for one-parameter semigroups, Proc. Amer. Math. Soc. 25 (1970), 488-494. MR 41 \# 4296.

9. — Quasi-analyticity and semigroups, Bull. Amer. Math. Soc. (6) 78 (1972), 909-922.

DEPARTMENT OF MATHEMATICS, UNIVERSITY OF WISCONSIN, MADISON, WISCONSIN 53706 